\title{
Test Scheduling for Multi-Clock Domain SoCs under Power Constraint
}

\author{
Tomokazu YONEDA $^{\dagger a}$, Member, Kimihiko MASUDA $^{\dagger *}$, Nonmember, and Hideo FUJIWARA ${ }^{\dagger b)}$, Fellow
}

SUMMARY This paper presents a power-constrained test scheduling method for multi-clock domain SoCs that consist of cores operating at different clock frequencies during test. In the proposed method, we utilize virtual TAM to solve the frequency gaps between cores and the ATE. Moreover, we present a technique to reduce power consumption of cores during test while the test time of the cores remain the same or increase a little by using virtual TAM. Experimental results show the effectiveness of the proposed method.

key words: multi-clock domain SoC, test scheduling, test access mechanism, power consumption

\section{Introduction}

Today's SoCs embed hundreds of memory cores and several different types of logic cores obtained from various vendors. Moreover, multiple clocks operate at multiple frequencies in a single SoC. Testing of SoCs is a crucial and time consuming problem due to the increasing design complexity. SoCs are increasingly tested in a modular fashion because the system integrator in most cases has very limited knowledge about the structural content of the adopted core, and hence deals with it as a black box. Therefore he/she cannot develop the DFT structures and the corresponding test patterns for it. This is especially true if a core is a hard one or is an encrypted Intellectual Property block [1]. In order to enable modular test, each embedded core should be isolated from its surrounding circuitry. Zorian et al. introduced a generic test architecture that enables modular test for SoCs [1]. It consists of the following three components: 1) test pattern source and test response sink, 2) test access mechanism (TAM), and 3) wrapper. The TAM propagates test patterns for a core from test pattern source to the core, and furthermore propagates the responses from the core to test pattern sink. The wrapper provides an interface between TAM and core, and also provides functions for cores to switch the mode of the cores: 1) normal, 2) INTEST (to test cores), 3) EXTEST (to test interconnects between cores), and 4) BYPASS defined in IEEE std. 1500 [2]. The goal is to develop techniques for wrapper design, TAM design and test schedule that minimizes test

Manuscript received April 9, 2007.

Manuscript revised August 3, 2007.

${ }^{\dagger}$ The authors are with Nara Institute of Science and Technology (NAIST),Ikoma-shi, 630-0192 Japan.

${ }^{*}$ Presently, the author is with SHARP Corporation, Tenri-shi, 632-8567 Japan.

a)E-mail: yoneda@is.naist.jp

b)E-mail: fujiwara@is.naist.jp

DOI: 10.1093/ietisy/e91-d.3.747 application time under given constraints such as the number of test pins and power consumption. A number of approaches have addressed wrapper design [3]-[5] which are IEEE std. 1500 compliant. Similarly, several TAM architectures have been proposed such as TestBus [6], [7], TESTRAIL [8], transparency based TAM [9]-[11]. Moreover, many approaches for core-internal test scheduling problem have been proposed [3], [8], [12]-[16]. Recently, [17] proposed a test scheduling method to minimize the overall test time for core-internal logic and core-external interconnects.

However, these previous approaches are applicable only to single-clock domain SoCs that consist of embedded cores operating at the same clock frequency during test. Today's SoC designs in telecommunications, networking and digital signal processing applications consist of embedded cores operating with different clock frequencies. The clock frequency of some embedded cores during test is limited by its scan chain frequencies. On the other hand, other cores may be testable at-speed in order to increase the coverage of non-modeled and performance-related defects. Moreover, there also exists a frequency gap between each embedded core and ATE used to test the SoC. From these facts, we can say that the previous approaches have the following two problems: 1) when test clock frequency of a core is higher than that of ATE, the ATE cannot provide test sequences at the same speed of the test clock frequency of the core, and 2) when test clock frequency of a core is lower than that of ATE, testing of the core by lowering the frequency of ATE does not make use of ATE capability effectively. Therefore, it is necessary to develop a technique that can solve the above problems for the multi-clock domain SoCs.

Recently, virtual TAM based on bandwidth matching [18] has been proposed in [19] to increase ATE capability when the clock frequency of a core is lower than that of ATE. Xu and Nicolici extended the virtual TAM technique to the multi-frequency TAM design to reduce the test time for the single-clock domain SoCs in [20]. Moreover, a wrapper design for cores with multiple clock domains was proposed in [21]-[24] to achieve at-speed testing of the cores by using virtual TAM technique. However, the test scheduling problem for the multi clock domain SoCs is not addressed in these literatures.

To the best of our knowledge, this paper gives a first discussion and a formulation of the core-internal test scheduling problem for multi-clock domain SoCs. We present a wrapper and TAM design for multi-clock domain SoCs and propose a test scheduling algorithm to minimize 
test time under power constraint. In the proposed method, we use virtual TAM for each core to solve a frequency gap between each core and a given ATE while the approach in [20] uses a virtual TAM for each test bus (i.e., all the cores assigned to the same test bus must be tested at the same frequency). Therefore, the proposed method in this paper has more flexibility for the test scheduling. Moreover, we also use virtual TAM in order to reduce the power consumption of the cores during test while the test time of the cores remain the same or increase a little. Therefore, the proposed method is effective for the power-constrained test scheduling. Experimental results show the effectiveness of the proposed method.

The rest of this paper is organized as follows. We discuss the power consumption and multi-clock domain SoCs in Sect. 2. Section 3 shows a power-conscious virtual TAM technique. After formulating a test scheduling problem for multi-clock domain SoCs in Sect.4, we present a powerconstrained test scheduling algorithm in Sect. 5. Experimental results are discussed in Sect. 6. Finally, Sect. 7 concludes this paper.

\section{Preliminaries}

\subsection{Power Consumption}

Power consumption in CMOS circuits can be classified into two categories: static power and dynamic power. Static power dissipation is caused by leakage or other current drawn continuously from the power supply. On the other hand, Dynamic power dissipation is caused by output switching. For the current CMOS technology, dynamic power is the dominant source of power consumption. High average power consumption causes structural damage to the silicon, bonding wires or package. And if peak power consumption exceeds a certain limit, designers cannot guarantee that the entire circuit will function correctly. It is said that average power consumption is closely related to scan operation while peak power consumption is related to capture operation during test. In this paper, we only consider the power consumption during scan operation defined as follows.

First, the energy $E(k)$ consumed in the circuit on application of consecutive two test vectors $\left(V_{k-1}, V_{k}\right)$ is defined as follows [25].

$$
E(k)=1 / 2 \cdot c_{0} \cdot V_{D D}^{2} \cdot \sum_{i} \cdot S_{i}(k) \cdot F_{i}
$$

where $c_{0}$ is the circuit's minimum parasitic capacitance, $V_{D D}$ is the power supply voltage, $S_{i}(k)$ is the number of switchings provoked by $V_{k}$ at node $i$, and $F_{i}$ is the number of fanout at node $i$. Let $N$ be the number of clock cycles for scan operation. The total energy consumed in the circuit during the scan operation is defined as follows.

$$
E_{\text {total }}=1 / 2 \cdot c_{0} \cdot V_{D D}^{2} \cdot \sum_{k=1}^{N} \sum_{i} \cdot S_{i}(k) \cdot F_{i}
$$

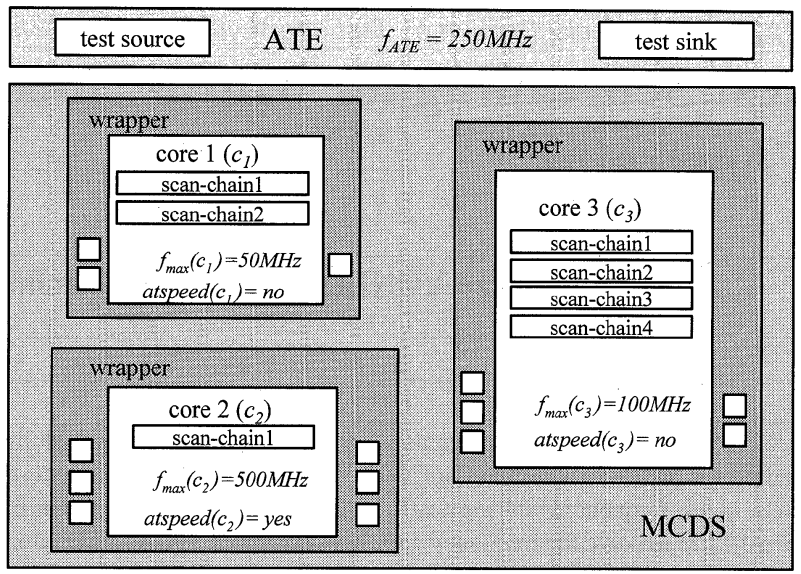

Fig.1 Multi-clock domain SoC.

Let $T$ be the clock period during scan operation (i.e., a fixed value for $T$ is used during scan operation). Then, the peak power $P_{\text {peak }}$ corresponds to the highest energy consumed during one clock period divided by $T$ as follows.

$$
P_{\text {peak }}=\max _{k}(E(k)) / T
$$

The average power $P_{\text {ave }}$ corresponds to the total energy divided by the scan shift time as follows.

$$
P_{\text {ave }}=E_{\text {total }} /(N \cdot T)
$$

The scan frequency $f$ is defined by $f=1 / T$. Therefore, power consumption during scan operation is proportional to the scan frequency.

\subsection{Multi-Clock Domain SoCs}

This section describes the formal notation we use to model the multi-clock domain SoC under test. An example of an $\mathrm{SoC}$ is shown in Fig. 1 where each core is wrapped to ease test access. Test pattern source and test response sink are implemented off-chip as an ATE. We assumed that an SoC consists of scan-designed cores and each core has single clock frequency during scan operation (i.e., single clock frequency during test data propagation from ATE to the core and from the core to ATE). This assumption is practical even for cores with multiple clock domains. For example, in [21], [26], the authors use single clock frequency during scan in/out operations while multiple clocks are used during capture operation to test delay faults in the circuits with multiple clock domains. In multi-clock domain SoCs, the clock frequency during scan in/out operation for a core can be different from that for other cores because each core has its own scan chain design and the requirement and limitation for the scan in/out frequency can be different from others. In this paper, we consider multi-clock domain SoCs where each core has single clock frequency during scan in/out operation while the frequency during scan in/out operation can be different between cores. In the sequel of this paper, we use the term "test frequency" as the clock frequency during 
scan in and out. The multi-clock domain SoC can be modeled as $M C D S=\left(C, P_{\max }\right)$ where:

$P_{\max }:$ maximum allowed power consumption;

$C=\left\{c_{1}, c_{2}, \ldots, c_{n}\right\}$ is a set of cores;

Each core $c_{i} \in C$ is characterized by

- $f_{\max }\left(c_{i}\right)$ : maximum test (scan) frequency;

- $\operatorname{power}\left(c_{i}\right)$ : power consumption at $f_{\max }\left(c_{i}\right)$;

- atspeed : at-speed test requirement;

- $R_{i}=\left\{r_{i 1}, r_{i 2}, \ldots\right\}$ is a set of wrapper designs; Each wrapper design $r_{i j} \in R_{i}$ is characterized by :

- $p i n\left(r_{i j}\right):$ number of pins required to test;

- $\operatorname{cycle}\left(r_{i j}\right)$ : number of clock cycles required to test;

For each core, a maximum test frequency and a power consumption at the frequency are given. Each core also has an information about the requirement of at-speed testing. atspeed $\left(c_{i}\right)=$ yes means that $c_{i}$ must be scanned in/out at $f_{\max }\left(c_{i}\right)$ (i.e., the test frequency affects test quality, and we cannot change it for test scheduling). atspeed $\left(c_{i}\right)=$ no means that $c_{i}$ can be scanned in/out at $f_{\max }\left(c_{i}\right)$ or lower frequencies (i.e., the test frequency does not affect test quality, and we can decrease it for test scheduling). Moreover, each core has a wrapper list that consists of possible wrapper designs for the core. Each wrapper design has the number of test pins and the number of clock cycles required to test the core with the wrapper design. The test time for $c_{i}$ operating at $f_{\max }\left(c_{i}\right)$ can be calculated as $\operatorname{cycle}\left(c_{i}\right) / f_{\max }\left(c_{i}\right)$.

\section{Virtual TAM for Power Minimization}

In multi-clock domain SoCs, there exist clock frequency gaps between the given ATE and cores during test. The frequency gaps between ATE and cores can be solved by using virtual TAM techniques [19] based on bandwidth matching [18]. Virtual TAMs are based on the following equation between the TAM width and operating frequency.

$$
W_{A T E}^{c_{i}} \cdot f_{A T E}=W_{T A M}^{c_{i}} \cdot f\left(c_{i}\right)
$$

where $W_{A T E}^{c_{i}}$ and $W_{T A M}^{c_{i}}$ are the ATE channel width and the TAM width assigned to core $c_{i}$, respectively, and $f_{A T E}$ and $f\left(c_{i}\right)$ are the ATE channel frequency and core test frequency (= virtual TAM frequency) respectively. When $f\left(c_{i}\right)$ is higher than $f_{A T E}$ shown in Fig. 2 (a), we insert a test data multiplexing (TDM) circuit between ATE outputs and the core inputs, and multiplex $\left\lceil f\left(c_{i}\right) / f_{A T E}\right\rceil \cdot m$ TAM wires at $f_{A T E}$ into $m$ virtual TAM wires at $f\left(c_{i}\right)$. On the other hand, when $f\left(c_{i}\right)$ is lower than $f_{A T E}$ shown in Fig. 2 (b), we insert a test data de-multiplexing (TDdeM) circuit between ATE output and the core inputs, and de-multiplex $n$ TAM wires at $f_{A T E}$ into $\left\lfloor n \cdot f_{A T E} / f\left(c_{i}\right)\right\rfloor$ virtual TAM wires at $f\left(c_{i}\right)$. To observe test responses, we need to insert TDM/TDdeM between the core output and ATE inputs in the similar fashion.

In [19], it is observed that virtual TAMs have the following two characteristics. First, TDM and TDdeM are implemented using parallel-in/serial- out registers at the inputs of the cores and serial-in/parallel-out registers at the outputs of the cores. Therefore, the hardware cost is relatively low compared to the area of the core itself. Second, since the TDM and TDdeM used for implementation are placed next to the cores, only the original TAM wires are routed through the SoC. Thus, the routing cost is also low.

In this paper, we also utilize the virtual TAM technique to reduce power consumption of a core while the test time remains the same or increases a little. From the Eqs. (3) and (4), we observe that the power consumption of a core during test can be reduced by lowering its test frequency. However, this increases test time of the core proportionally to the power reduction ratio. Here, we insert TDdeM/TDM circuits between the ATE and the core. Then, more virtual TAM wires become available for the core, and test time can be reduced. In the best case, we can reduce the power

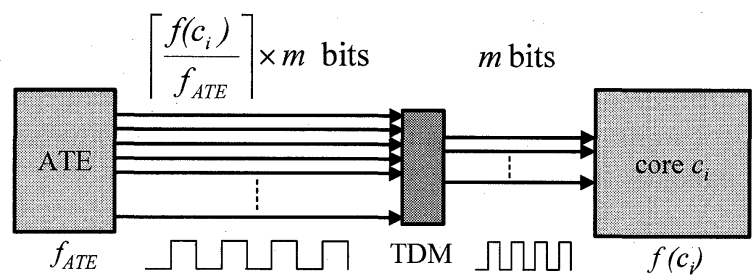

(a) $f_{A T E}<f\left(c_{i}\right)$.

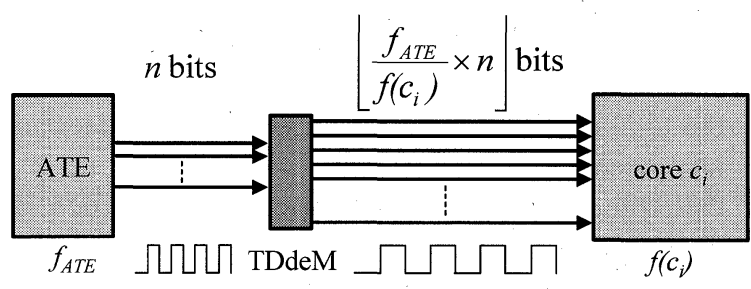

(b) $f_{A T E}>f\left(c_{i}\right)$

Fig. 2 Test data multiplexing/de-multiplexing.

Table 1 An example of power-conscious virtual TAM for core23 in p93791 $\left(f_{\text {ATE }}=200 \mathrm{MHz}\right)$.

\begin{tabular}{|c|c|c|c|c|c|}
\hline \multirow[b]{2}{*}{$\begin{array}{l}\text { TAM } \\
\text { (bits) }\end{array}$} & \multicolumn{2}{|c|}{$\begin{array}{l}f(\text { core } 23) \\
=200 \mathrm{MHz}\end{array}$} & \multicolumn{2}{|c|}{$\begin{array}{l}f(\text { core } 23) \\
=100 \mathrm{MHz}\end{array}$} & \multirow{2}{*}{$\begin{array}{c}\text { test time } \\
\text { increase } \\
(\%)\end{array}$} \\
\hline & $\begin{array}{l}\text { VTAM } \\
\text { (bits) }\end{array}$ & $\begin{array}{l}\text { time } \\
(\mu \mathrm{s})\end{array}$ & $\begin{array}{l}\text { VTAM } \\
\text { (bits) }\end{array}$ & $\begin{array}{l}\text { time } \\
(\mu \mathrm{s})\end{array}$ & \\
\hline 1 & 1 & 9184.59 & 2 & 9185.95 & 0.01 \\
\hline 2 & 2 & 4592.98 & 4 & 4754.04 & 3.51 \\
\hline 3 & 3 & 3063.09 & 6 & 3177.19 & 3.72 \\
\hline 4 & 4 & 2377.02 & 8 & 2408.74 & 1.33 \\
\hline 5 & 5 & 1838.79 & 10 & 2006.89 & 9.14 \\
\hline 6 & 6 & 1588.60 & 12 & 1607.39 & 1.18 \\
\hline 7 & 7 & 1387.67 & 14 & 1569.79 & 13.12 \\
\hline 8 & 8 & 1204.37 & 16 & 1205.54 & 0.10 \\
\hline 9 & 9 & 1022.20 & 18 & 1205.54 & 17.94 \\
\hline 10 & 10 & 1003.45 & 20 & 1203.19 & 19.91 \\
\hline 11 & 11 & 972.90 & 22 & 1142.09 & 17.39 \\
\hline 12 & 12 & 803.70 & 24 & 806.04 & 0.29 \\
\hline 13 & 13 & 802.52 & 26 & 806.04 & 0.44 \\
\hline 14 & 14 & 784.90 & 28 & 806.04 & 2.69 \\
\hline 15 & 15 & 614.49 & 30 & 806.04 & 31.17 \\
\hline 16 & 16 & 602.77 & 32 & 803.69 & 33.33 \\
\hline
\end{tabular}


consumption for a core without an increase in test time by using the above power-conscious virtual TAM technique. In this paper, we assume that the power consumption of TDM/TDdeM circuits is negligible from the above observation in [19].

Table 1 shows an example of the power-conscious virtual TAM. In this example, we consider the wrapper design for core23 in p93791 from ITC'02 SoC benchmarks [27]. Columns "time" show the test time for the core when the clock frequency of ATE is $200 \mathrm{MHz}$. For each TAM width, we can achieve $50 \%$ power reduction by decreasing the frequency from $200 \mathrm{MHz}$ to $100 \mathrm{MHz}$. On the other hand, test time increases by only a few percent in some cases while the other cases increase test time by 10 to $34 \%$. We can observe similar trends for other cores in other SoC. Therefore, for power-constrained test scheduling, test time can be reduced if we select a test frequency for each core effectively.

\section{Problem Formulation}

We formulate the power-constrained test scheduling problem for multi-clock domain SoCs $P_{m c d s}$ that we address in this paper as follows.

Definition 1: $P_{m c d s}$ : Given a multi-clock domain SoC $M C D S$, the number of available test pins $W_{\max }$ and the clock frequency of ATE $f_{A T E}$, determine a wrapper design $r_{i}^{\text {test }}$ and test frequency $f\left(c_{i}\right)$ for each core $c_{i}$ and a test schedule such that

1. the total number of test pins used at any moment does not exceed $W_{\max }$,

2. the total power consumption used at any moment does not exceed $P_{\max }$,

3. each core satisfies at-speed test requirement (i.e., if atspeed $\left(c_{i}\right)=$ yes, $c_{i}$ must be tested at $f_{\max }\left(c_{i}\right)$. Otherwise, $c_{i}$ can be tested at frequencies lower than $\left.f_{\max }\left(c_{i}\right)\right)$, and

4. the overall SoC test time is minimized.

\section{Scheduling Algorithm}

This section presents a heuristic algorithm for $P_{m c d s}$. The outline of the proposed algorithm is shown in Fig. 3.

\section{Step 1: Testability Analysis}

First, the algorithm checks whether there is a solution for a given problem instance (Line 1). For a core $c_{i}$ such that atspeed $\left(c_{i}\right)=$ yes, we cannot change the test frequency $f_{\max }\left(c_{i}\right)$ and power consumption $\operatorname{power}\left(c_{i}\right)$ during test. Therefore, there is no solution under the given $P_{\max }$ if power $\left(c_{i}\right)$ exceeds $P_{\max }$. Moreover, we have no solution if the ATE cannot provide enough bandwidth for $c_{i}$ to test at $f_{\max }\left(c_{i}\right)$. Now, we summarize the conditions as follows.

For each $c_{i} \in C$ such that atspeed $\left(c_{i}\right)=y e s$, if $c_{i}$ cannot satisfy the following both two conditions, there is no solution and the algorithm exits. Otherwise, it moves to Step 2.

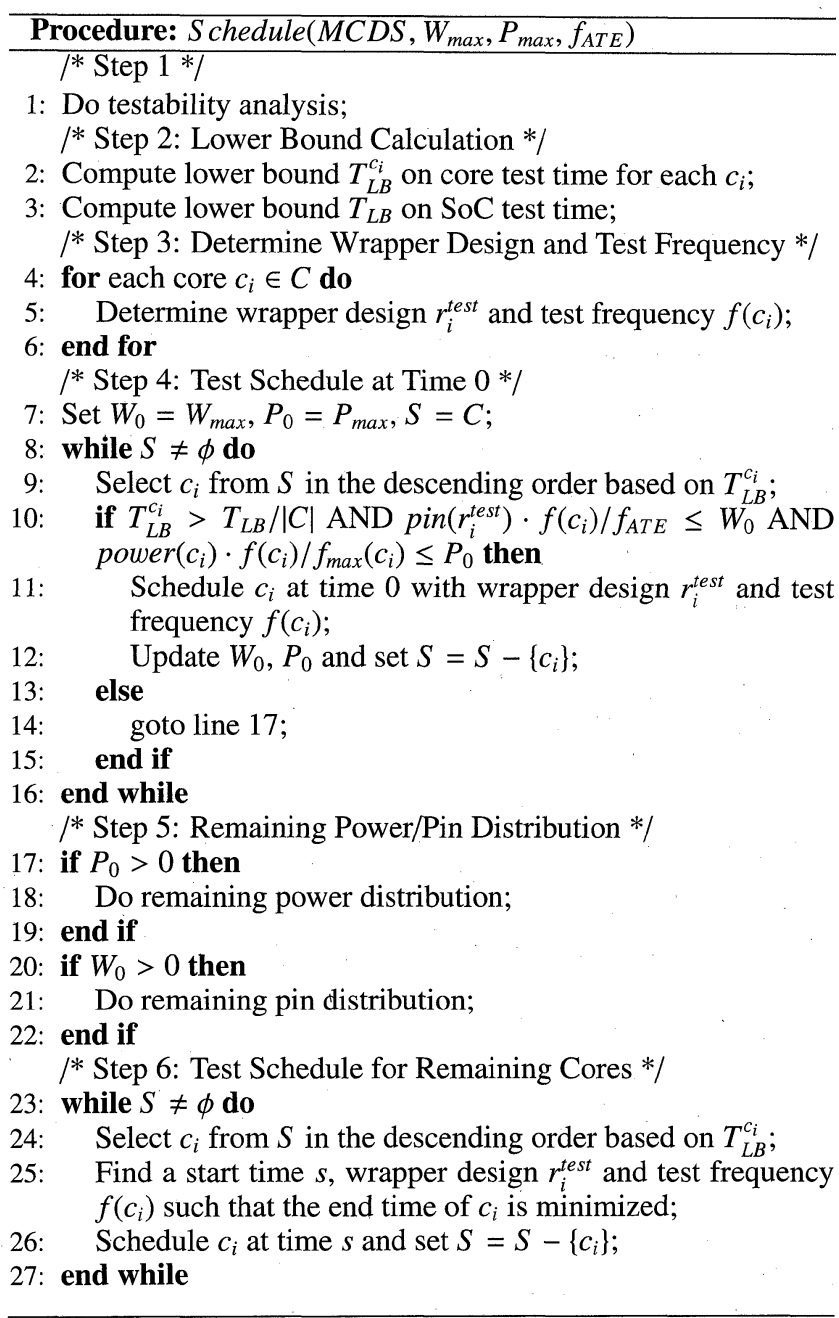

Fig. 3 Outline of the proposed algorithm.

$$
\begin{aligned}
& P_{\text {max }} \geq \operatorname{power}\left(c_{i}\right), \text { and } \\
& W_{\text {max }} \cdot f_{A T E} \geq \min _{j}\left\{\operatorname{pin}\left(r_{i j}\right)\right\} \cdot f_{\max }\left(c_{i}\right)
\end{aligned}
$$

\section{Step 2: Lower Bound Calculation}

The authors in [8] proposed an architecture independent lower bounds on core and SoC test time. In this step (Line 2-3), similar lower bounds are calculated for use in the later steps. First, we calculate a lower bound $T_{L B}^{c_{i}}$ on test time of each core $c_{i}$ as follows.

$$
T_{L B}^{c_{i}}=\frac{\operatorname{cycle}\left(r_{i}^{\max }\right)}{f_{\max }\left(c_{i}\right)}
$$

where $r_{i}^{\max }$ is the wrapper configuration of $c_{i}$ such that $\operatorname{pin}\left(r_{i}^{\max }\right)$ is maximum and $r_{i}^{\max }$ satisfies the following condition.

$$
\operatorname{pin}\left(r_{i}^{\max }\right) \cdot f_{\max }\left(c_{i}\right) \leq W_{\max } \cdot f_{\text {ATE }}
$$

Then, we calculate a lower bound $T_{L B}$ on SoC test time as follows. 


$$
T_{L B}=\max \left(\max _{i}\left\{T_{L B}^{c_{i}}\right\}, \frac{\text { TotalData }}{W_{\max } \cdot f_{A T E}}\right)
$$

where TotalData $=\sum_{i} \operatorname{pin}\left(r_{i}^{\min }\right) \cdot \operatorname{cycle}\left(r_{i}^{\min }\right)$ and $r_{i}^{\min }$ is the wrapper configuration of $c_{i}$ such that $\operatorname{pin}\left(r_{i}^{\min }\right)$ is minimum.

\section{Step 3: Determine Wrapper Design and Test Frequency} Main idea in this step (Line 4-6) is to lower the test frequencies of cores which are not required to test at-speed in order to increase test concurrency for power-constrained test scheduling in the next step. For each core $c_{i}$, we determine a wrapper design $r_{i}^{\text {test }}$ and an integer frequency division factor $m_{c_{i}}$ such that

(i) $T_{L B} \geq \operatorname{cycle}\left(r_{i}^{\text {test }}\right) \cdot \frac{m_{c_{i}}}{f_{\max }\left(c_{i}\right)}$,

(ii) $W_{\max } \cdot f_{A T E} \geq \operatorname{pin}\left(r_{i}^{\text {test }}\right) \cdot \frac{f_{\max }\left(c_{i}\right)}{m_{c_{i}}}$,

(iii) $P_{\max } \geq \operatorname{power}\left(c_{i}\right) \cdot \frac{f_{\max }\left(c_{i}\right)}{m_{c_{i}}}$,

(iv) $m_{c_{i}}=1$ if atspeed $\left(c_{i}\right)=$ yes, otherwise, $m_{c_{i}}$ is maximized, and

(v) $\operatorname{pin}\left(r_{i}^{\text {test }}\right)$ is minimized subject to (iv).

Test frequency $f\left(c_{i}\right)$ for $c_{i}$ is determined as follows.

$$
f\left(c_{i}\right)=\frac{f_{\max }\left(c_{i}\right)}{m_{c_{i}}}
$$

In this paper, we assume that the frequency division factor $m_{c_{i}}$ is an integer. However, to simplify the hardware implementation we can limit $m_{c_{i}}$ as two's exponent or userspecified frequency set.

\section{Step 4: Test Schedule at Time 0}

This step determines test schedule at time 0 (Line 7-16). First, we initialize the available power consumption $P_{0}$, available test pins $W_{0}$ at time 0 and the set of unscheduled cores $S$ (Line 7). Then, we sort cores in the descending order based on $T_{L B}^{c_{i}}$ (Line 9). After that, we schedule a core $c_{i}$ in the above order at time 0 with wrapper $r_{i}^{\text {test }}$ and test frequency $f\left(c_{i}\right)$ (Line 11), and update the corresponding variables (Line 12). This process is repeated until all cores are scheduled or $c_{i}$ cannot satisfy the conditions at Line 10 . The condition $T_{L B}^{c_{i}}>T_{L B} /|C|$ can prevent us from scheduling cores with small amount of test data to time 0 . Instead of scheduling such small cores at time 0 , next step tries to reduce the test time of the cores scheduled in this step by distributing the remaining available power and test pins.

Figure 4 shows a current test schedule generated after Step 4. In Fig. 4 (a), the horizontal axis denotes the test time, and the vertical axis denotes the power consumption used in each test time. In Fig. 4 (b), the horizontal axis denotes the test time, and the vertical axis denotes the number of test pin used in each test time.

\section{Step 5: Remaining Power/Pin Distribution at Time 0}

There exists a case where $P_{0}$ (available power consumption at time 0) does not reach 0 after Step 4 as shown in Fig. 4 (a). This is because Step 4 terminates when one of the three conditions in Line 10 cannot be satisfied. In this step (Line 17-19), we find a core $c_{i}$ with longest test time among the currently scheduled cores such that

$$
\begin{aligned}
& \text { (i) } m_{c_{i}} \geq 2 \\
& \text { (ii) } P_{0} \geq \operatorname{power}\left(c_{i}\right) \cdot\left(\frac{1}{m_{c_{i}}-1}-\frac{1}{m_{c_{i}}}\right) \text {, and } \\
& \text { (iii) } \frac{P_{\max }}{2} \geq \frac{\operatorname{power}\left(c_{i}\right)}{m_{c_{i}}-1}
\end{aligned}
$$

If there exists such a core $c_{i}$, we update $m_{c_{i}}$ to $m_{c_{i}}-1$, and reduce the test time of $c_{i}$ by increasing $f\left(c_{i}\right)$ according to Eq. (11) while satisfying power constraint by Eq. (13). Equation (14) can prevent one core from dominating power consumption, and help us to increase the test concurrency when the remaining cores are scheduled in next step (Step 6). This process is repeated while both of the following two conditions are satisfied.

1. $P_{0}>0$, and

2. there exists a core that satisfies all three Eqs. (12), (13) and (14).

Figure 5 (a) shows a result where we apply this process to the current schedule generated after Step 4 shown in Fig. 4. In this example, frequencies for core 2, 3, 4 and 6 are increased. Consequently, the test time for these cores are reduced.

Similarly, there exists a case where $W_{0}$ (available test

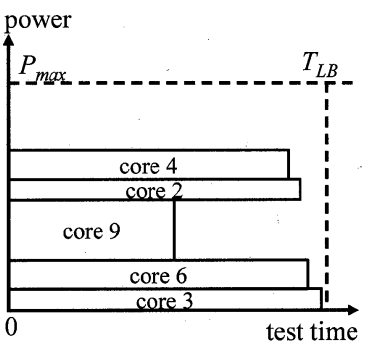

(a) Power vs test time.

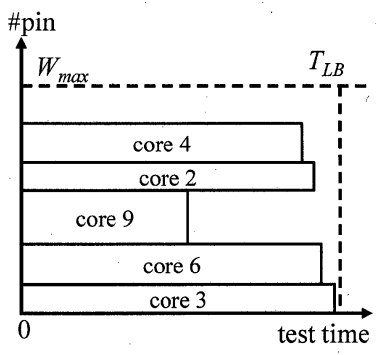

(b) Pin vs test time.
Fig. 4 Test schedule after Step 4.

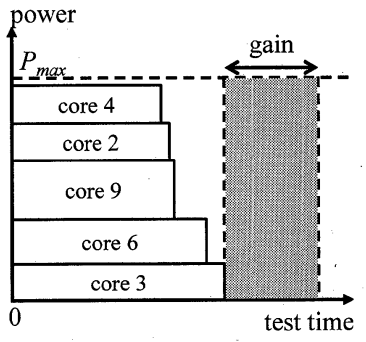

(a) Power vs test time after recalculating test frequencies.

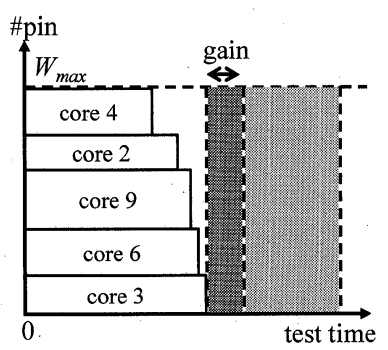

(b) Pin vs test time after redesign wrappers.
Fig.5 Test schedule after Step 5. 
pins at time 0 ) does not reach 0 after Step 4 . In this case, we find a core $c_{i}$ with longest test time, then assign 1 test pin to $c_{i}$ and reduce the test time. This process is repeated while $W_{0}>0$ (Line 20-22). Figure 5 (b) shows a result where we apply this process to the current schedule corresponding to Fig. 5 (a).

\section{Step 6: Test Scheduling for Remaining Cores}

This step determines test schedules for the remaining unscheduled cores based on BFD heuristic. First, we pick a core $c_{i}$ in the descending order based on $T_{L B}^{c_{i}}$ (Line 24). Then, we find a start time, wrapper design $r_{i}^{\text {test }}$ and test frequency $f\left(c_{i}\right)$ for $c_{i}$ such that the end test time of $c_{i}$ is minimized as follows (Line 25).

1. Let $S$ be a set of start time candidates that consists of the end time of scheduled cores in the current schedule. For each candidate $s \in S$, we calculate available power consumption $P_{s}$ and available test pin $W_{s}$ from the current schedule.

2. For each candidate $s \in S$,

a. Select a highest test frequency $f_{s}\left(c_{i}\right)$ such that

$$
\text { (i) } \operatorname{power}\left(c_{i}\right) \cdot \frac{f_{s}\left(c_{i}\right)}{f_{\max }\left(c_{i}\right)} \leq P_{s} \text {. }
$$

b. Select a wrapper design $r_{i, s}^{\text {test }}$ such that

(i) $\operatorname{pin}\left(r_{i, s}^{t e s t}\right) \cdot f_{s}\left(c_{i}\right) \leq W_{s} \cdot f_{A T E}$ and,

(ii) $\operatorname{pin}\left(r_{i, s}^{\text {test }}\right)$ is maximized.

c. Calculate the end time $t_{i, s}$ when $c_{i}$ starts its test at time $s$ with wrapper $r_{i, s}^{\text {test }}$ at frequency $f_{s}\left(c_{i}\right)$.

3. Schedule $c_{i}$ at time $s$ with wrapper $r_{i, s}^{\text {test }}$ at frequency $f_{s}\left(c_{i}\right)$ such that

(i) $t_{i, s}$ is minimized,

(ii) the test of $c_{i}$ does not overlap the tests of cores already scheduled in the current schedule.

Figure 6 shows an example of the test scheduling for core 5. Here, a set of start time candidates $S$ consists of five elements: $s_{1}, s_{2}, s_{3}, s_{4}, s_{5}$. For each candidate $s \in S$, we calculate a end time $t_{5, s}$ by determining a test frequency $f_{s}\left(c_{5}\right)$

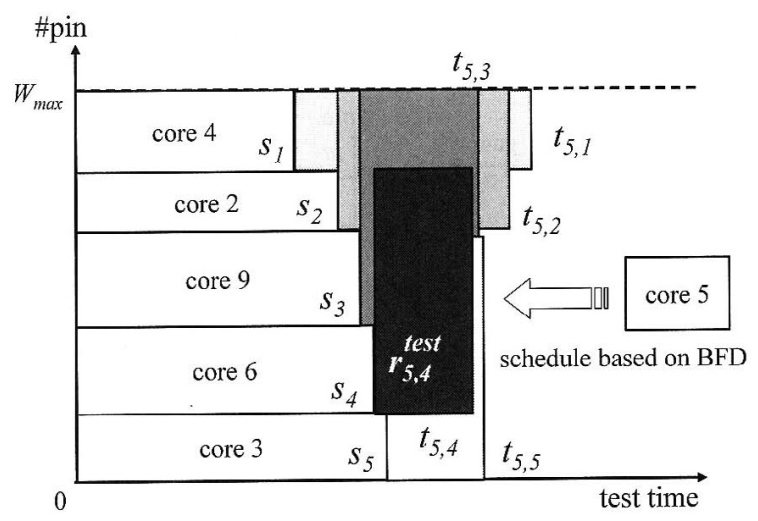

Fig. 6 An example of test scheduling for core 5. and a wrapper design $r_{5, s}^{\text {test }}$ shown as a rectangle in Fig. 6. In this example, core 5 is scheduled to start its test at time $s_{4}$ with a wrapper $r_{5,4}^{\text {test }}$ at frequency $f_{4}\left(c_{5}\right)$ since the end time $t_{5,4}$ has a minimum value.

This process is repeated until all the remaining cores are scheduled (Line 23-27). Finally, we can get a complete test schedule.

\section{Experimental Results}

In Sect. 6.1, we show experimental results for multi-clock domain SoCs with power constraints. Section 6.2 presents experimental results for single-clock domain SoCs with power constraints ("d695" and "h953" from ITC'02 SoC benchmarks [27] because only these two SoCs have power information in the benchmarks) in order to show the effectiveness of our approach compared to previous works. All the experimental results can be obtained within $0.1 \mathrm{sec}$. on a SunBlade 2000 workstation ( $1.05 \mathrm{GHz}$ with $8 \mathrm{~GB}$ RAM).

\subsection{Results for Multi-Clock Domain SoCs}

Since there exists no approach that has tackled the test scheduling problem for multi-clock domain SoCs, it is difficult to compare with previous works. We have decided to analyze the trade-offs of the proposed method in terms of the number of available test pin, the clock frequency of ATE, maximum allowed power consumption and test time for two multi-clock domain SoCs. Table 2 shows the multiclock domain SoC $M C D S_{1}$ used in this experiment. This SoC consists of 14 cores. First 10 cores are from "d695" in ITC'02 SoC benchmarks [27]. "flexible ( $\geq 2)$ " in column "wrapper list" denotes that we can design any wrapper (wrapper with any number of test pins) by the procedure proposed in [3], [4]. We use the same power consumption shown in [14], and assume that $f_{\max }\left(c_{i}\right)=50 \mathrm{MHz}$ and atspeed $\left(c_{i}\right)=n o$ for these 10 cores. The wrappers for core 11 and core 12 are already designed (i.e., 64 wrapper pins and 472 test cycles for core 11,32 wrapper pins and 782 test cycles for core 12 , respectively). We assume that these two

Table 2 An multi-clock domain SoC $M C D S_{1}$.

\begin{tabular}{c|c|c|r|r}
\hline core & $\begin{array}{c}\text { at-speed } \\
\text { requirement }\end{array}$ & $\begin{array}{c}\text { wrapper list } \\
\text { (pins) }\end{array}$ & $\begin{array}{r}\text { test freq. } \\
\text { (MHz) }\end{array}$ & $\begin{array}{r}\text { powcr } \\
\text { (unit) }\end{array}$ \\
\hline \hline 1 & no & flexible( $\geq 2)$ & 50 & 660 \\
\hline 2 & no & flexible $(\geq 2)$ & 50 & 602 \\
\hline 3 & no & flexible( $\geq 2)$ & 50 & 823 \\
\hline 4 & no & flexible $(\geq 2)$ & 50 & 275 \\
\hline 5 & no & flexible( $(\geq 2)$ & 50 & 690 \\
\hline 6 & no & flexible( $(\geq 2)$ & 50 & 354 \\
\hline 7 & no & flexible $(\geq 2)$ & 50 & 530 \\
\hline 8 & no & flexible $(\geq 2)$ & 50 & 753 \\
\hline 9 & no & flexible $(\geq 2)$ & 50 & 641 \\
\hline 10 & no & flexible $(\geq 2)$ & 50 & 1144 \\
\hline 11 & yes & fixed $(64)$ & 100 & 480 \\
\hline 12 & yes & fixed $(32)$ & 200 & 940 \\
\hline 13 & no & flexible $(\geq 2)$ & 20 & 212 \\
\hline 14 & no & flexible $(\geq 2)$ & 25 & 345 \\
\hline
\end{tabular}


Table 3 Test time results $[\mu \mathrm{s}]$ for multi-clock domain $\operatorname{SoC} M C D S_{1}\left(1 \leq m_{c_{i}} \leq 8\right)$.

\begin{tabular}{|c|c|c|c|c|c|c|c|c|c|c|}
\hline \multirow[b]{3}{*}{$f_{A T E}$} & \multirow[b]{3}{*}{$P_{\max }$} & \multicolumn{9}{|c|}{$W_{\max }$} \\
\hline & & \multicolumn{3}{|c|}{32 pin } & \multicolumn{3}{|c|}{64 pin } & \multicolumn{3}{|c|}{128 pin } \\
\hline & & $\begin{array}{c}\text { Case1 } \\
m_{c_{i}}= \\
\text { integer }\end{array}$ & $\begin{array}{c}\text { Case3 } \\
m_{c_{i}}=1 \\
(\text { diff1) }\end{array}$ & $\begin{array}{c}T_{L B} \\
\\
(\operatorname{diff} 1) \\
\end{array}$ & $\begin{array}{c}\text { Case1 } \\
m_{c_{i}}= \\
\text { integer }\end{array}$ & $\begin{array}{c}\text { Case3 } \\
m_{c_{i}}=1 \\
\text { (diff1) }\end{array}$ & $\begin{array}{c}T_{L B} \\
\text { (diff1) }\end{array}$ & $\begin{array}{c}\text { Case1 } \\
m_{c_{i}}= \\
\text { integer }\end{array}$ & $\begin{array}{c}\text { Case3 } \\
m_{c_{i}}=1 \\
\text { (diff1) }\end{array}$ & $\begin{array}{c}T_{L B} \\
(\text { diff1) } \\
\end{array}$ \\
\hline \multirow{3}{*}{$200 \mathrm{MHz}$} & 1000 & 637.13 & UT & & 632.41 & $\overline{\mathrm{UT}}$ & & 632.41 & UT & \\
\hline & 2000 & 325.29 & $\begin{array}{c}375.65 \\
(15.5 \%)\end{array}$ & & 301.08 & $\begin{array}{l}321.52 \\
(6.8 \%) \\
\end{array}$ & & 301.08 & $\begin{array}{l}321.52 \\
(6.8 \%)\end{array}$ & \\
\hline & 3000 & 312.09 & $\begin{array}{l}318.73 \\
(2.1 \%) \\
\end{array}$ & $\begin{array}{c}295.27 \\
(-5.4 \%) \\
\end{array}$ & 204.20 & $\begin{array}{l}209.42 \\
(2.6 \%)\end{array}$ & $\begin{array}{l}204.20 \\
(0.0 \%) \\
\end{array}$ & 204.20 & $\begin{array}{l}209.42 \\
(2.6 \%) \\
\end{array}$ & $\begin{array}{l}204.20 \\
(0.0 \%) \\
\end{array}$ \\
\hline \multirow{3}{*}{$100 \mathrm{MHz}$} & 1000 & UT & UT & & 637.13 & UT & & 632.41 & UT & \\
\hline & 2000 & UT & UT & & 325.29 & $\begin{array}{c}375.65 \\
(15.5 \%) \\
\end{array}$ & & 301.08 & $\begin{array}{l}321.52 \\
(6.8 \%)\end{array}$ & \\
\hline & 3000 & UT & UT & & 312.09 & $\begin{array}{l}318.73 \\
(2.1 \%) \\
\end{array}$ & $\begin{array}{c}295.27 \\
(-5.4 \%) \\
\end{array}$ & 204.20 & $\begin{array}{l}209.42 \\
(2.6 \%) \\
\end{array}$ & $\begin{array}{l}204.20 \\
(0.0 \%) \\
\end{array}$ \\
\hline \multirow{3}{*}{$50 \mathrm{MHz}$} & 1000 & UT & UT & & UT & UT & & 637.13 & UT & \\
\hline & 2000 & UT & UT & & UT & UT & & 325.29 & $\begin{array}{c}375.65 \\
(15.5 \%) \\
\end{array}$ & \\
\hline & 3000 & UT & UT & & UT & UT & & 312.09 & $\begin{array}{c}318.73 \\
(2.1 \%)\end{array}$ & $\begin{array}{c}295.27 \\
(-5.4 \%)\end{array}$ \\
\hline
\end{tabular}

Table 4 Test time results $[\mu \mathrm{s}]$ for $p 93791$ when $f_{A T E}=50 \mathrm{MHz}\left(1 \leq m_{c_{i}} \leq 8\right)$.

\begin{tabular}{|c|c|c|c|c|c|c|c|c|c|c|c|c|}
\hline \multirow[b]{3}{*}{$P_{\max }$} & \multicolumn{12}{|c|}{$W_{\max }$} \\
\hline & \multicolumn{4}{|c|}{32 pin } & \multicolumn{4}{|c|}{64 pin } & \multicolumn{4}{|c|}{128 pin } \\
\hline & $\begin{array}{c}\text { Case1 } \\
m_{c_{i}}= \\
\text { integer }\end{array}$ & $\begin{array}{c}\text { Case2 } \\
m_{c_{i}}=2^{x} \\
\text { (diff1) }\end{array}$ & $\begin{array}{c}\text { Case3 } \\
m_{c_{i}}=1 \\
\text { (diff1) }\end{array}$ & $\begin{array}{c}T_{L B} \\
(\operatorname{diff1})\end{array}$ & $\begin{array}{c}\text { Case1 } \\
m_{c_{i}}= \\
\text { integer }\end{array}$ & $\begin{array}{c}\text { Case2 } \\
m_{c_{i}}=2^{x} \\
\text { (diff1) }\end{array}$ & $\begin{array}{c}\text { Case3 } \\
m_{c_{i}}=1 \\
\text { (diff1) }\end{array}$ & $\begin{array}{c}T_{L B} \\
(\operatorname{diff} 1)\end{array}$ & $\begin{array}{c}\text { Case1 } \\
m_{c_{i}}= \\
\text { integer }\end{array}$ & $\begin{array}{c}\text { Case2 } \\
m_{c_{i}}=2^{x} \\
(\text { diff } 1)\end{array}$ & $\begin{array}{c}\text { Case3 } \\
m_{c_{i}}=1 \\
(\operatorname{diff} 1)\end{array}$ & $\begin{array}{c}T_{L B} \\
(\operatorname{diff} 1)\end{array}$ \\
\hline 15000 & 36385 & $\begin{array}{c}36430 \\
(0.1 \%) \\
\end{array}$ & UT & & 20381 & $\begin{array}{c}20506 \\
(0.6 \%)\end{array}$ & UT & & 9712 & $\begin{array}{c}10562 \\
(8.8 \%)\end{array}$ & $\overline{\mathrm{UT}}$ & \\
\hline 30000 & 36227 & $\begin{array}{c}36227 \\
(0.0 \%)\end{array}$ & $\begin{array}{c}36511 \\
(0.8 \%)\end{array}$ & & 19086 & $\begin{array}{c}19992 \\
(4.7 \%)\end{array}$ & $\begin{array}{c}20430 \\
(7.0 \%)\end{array}$ & & 9604 & $\begin{array}{c}10033 \\
(4.5 \%)\end{array}$ & $\begin{array}{c}10401 \\
(8.3 \%)\end{array}$ & \\
\hline 50000 & 36227 & $\begin{array}{c}36227 \\
(0.0 \%)\end{array}$ & $\begin{array}{c}36480 \\
(0.7 \%)\end{array}$ & $\begin{array}{c}34988 \\
(-3.4 \%)\end{array}$ & 18420 & $\begin{array}{c}19992 \\
(8.5 \%)\end{array}$ & $\begin{array}{c}20411 \\
(10.8 \%)\end{array}$ & $\begin{array}{c}17494 \\
(-5.0 \%)\end{array}$ & 9444 & $\begin{array}{c}9903 \\
(4.9 \%)\end{array}$ & $\begin{array}{c}10401 \\
(10.1 \%)\end{array}$ & $\begin{array}{c}8747 \\
(-7.4 \%)\end{array}$ \\
\hline
\end{tabular}

cores are tested at higher frequencies than other cores, and atspeed $\left(c_{i}\right)=$ yes. Core 13 and core 14 are copies of core 7 and core 5, respectively. However, we assume that these two cores are tested at lower frequencies than other cores.

Table 3 shows test time results when $f_{A T E}=200 \mathrm{MHz}$, $100 \mathrm{MHz}$ and $50 \mathrm{MHz}$ for $M C D S_{1}$. We did experiments for the following three cases with respect to the integer frequency divisor $m_{c_{i}}$ used in the proposed algorithm: (1) $m_{c_{i}}$ is integer, (2) $m_{c_{i}}$ is 2's exponent and (3) $m_{c_{i}}=1$ (i.e., it is the same as the case we set atspeed $\left(c_{i}\right)=$ yes for all cores). However, the results for Case2 are identical for Case1 and we did not include in the table. Column " $T_{L B}$ " denotes the power-independent theoretical lower bound on test time defined by Eq. (10). In this table, the test time results are shown as " $\mu$ sec." and the number in parentheses denotes the test time increase relative to Case1. "UT" denotes that there exists no solution for the given parameters. In this SoC, since core 11 should be tested at $100 \mathrm{MHz}$ with 64 pins, we observe that there exists no solution for three cases: 1) $f_{A T E}=100 \mathrm{MHz}$ and $\left.W_{\max }=32,2\right) f_{A T E}=50 \mathrm{MHz}$ and
$W_{\max }=32$, and 3) $f_{A T E}=50 \mathrm{MHz}$ and $W_{\max }=64$. We also observe that test time depends on the product of $f_{A T E}$ and $W_{\max }$. Therefore, when we use a high speed ATE, we can test SoCs with small number of test pins. On the other hand, even when we use a low speed ATE, we can achieve the same test time by using more test pins. From this results, the designer can decide the number of test pins and the speed of the test pin considering the total cost for them. Moreover, we can observe the effectiveness of lowering test frequencies by comparing Case 1 with Case 3 . We can obtain savings in test time up $15 \%$ by lowering test frequencies. The difference from power-independent lower bound $T_{L B}$ is at most only $5.4 \%$ when $P_{\max }=3000$. Therefore, we can say that the proposed heuristic algorithm is effective and efficient.

Table 4 shows the test time results for another multiclock domain SoC $p 93791$ from ITC'02 SoC benchmarks [27] when $f_{A T E}=50 \mathrm{MHz}$. As the original benchmark SoC do not have any data related to power consumption, we used the following settings for each core $c_{i}$ : (1) 
Table 5 Test time results (\# cycles) for single-clock domain SoCs.

\begin{tabular}{|c|c|c|c|c|c|c|c|c|c|c|}
\hline \multirow{3}{*}{$\mathrm{SoC}$} & \multirow{3}{*}{$P_{\max }$} & \multicolumn{9}{|c|}{$W_{\max }$} \\
\hline & & \multicolumn{3}{|c|}{32 pin } & \multicolumn{3}{|c|}{64 pin } & \multicolumn{3}{|c|}{128 pin } \\
\hline & & $3 \mathrm{D}[14]$ & EA [15] & proposed & $3 \mathrm{D}[14]$ & EA [15] & proposed & $3 \mathrm{D}[14]$ & EA [15] & proposed \\
\hline \multirow[t]{4}{*}{$\mathrm{d} 695$} & 1000 & NA & NA & 44528 & NA & NA & 27482 & NA & NA & 24707 \\
\hline & 1500 & 45560 & - & 42981 & 27573 & - & 22690 & 16841 & - & 16239 \\
\hline & 2000 & 43221 & - & 42632 & 24171 & - & 21838 & 14128 & - & 12753 \\
\hline & 2500 & 43221 & - & 42564 & 23721 & - & 21616 & 12993 & - & 11180 \\
\hline \multirow[t]{3}{*}{ h953 } & $5 \times 10^{9}$ & NA & NA & 119357 & NA & NA & 119357 & NA & NA & 119357 \\
\hline & $6 \times 10^{9}$ & 122636 & 122636 & $\mathbf{1 1 9 3 5 7}$ & 122636 & 122636 & 119357 & 122636 & 122636 & 119357 \\
\hline & $7 \times 10^{9}$ & 119357 & 119357 & 119357 & 119357 & 119357 & 119357 & 119357 & 119357 & 119357 \\
\hline
\end{tabular}

$f_{\text {max }}\left(c_{i}\right)=50 \mathrm{MHz},(2)$ atspeed $\left(c_{i}\right)=$ no and (3) power $\left(c_{i}\right)$ is the total number of scan FFs in $c_{i}$. If we limit $m_{c_{i}}$ to 2 's exponent, test time is increased up to $8.8 \%$. Test time is further increased up to $10.8 \%$ by limiting $m_{c_{i}}$ to 1 . Finally, the difference from $T_{L B}$ is at most only $7.4 \%$ when $P_{\max }=50000$, and it shows the effectiveness and efficiency of the proposed algorithm.

\subsection{Comparison with Other Approaches}

In order to show the effectiveness of our approach compared to previous works, we present experimental results for the single-clock domain SoCs with power constraint. We use "d695" and "h953" from ITC'02 SoC benchmarks [27] as the single-clock domain SoCs by assuming that $f_{A T E}=$ $50 \mathrm{MHz}$, and $f_{\text {max }}\left(c_{i}\right)=50 \mathrm{MHz}$ and atspeed $\left(c_{i}\right)=$ no for all core $c_{i} \in C$. This is because only these two SoCs have power information in the benchmarks (for "d695", we use the same power consumption shown in [14]). Table 5 shows the test time results of the proposed method and the previous power-constrained approaches [14], [15] which are applicable only to the single-clock domain SoCs. In this table, test time results are shown as the number of clock cycles at $50 \mathrm{MHz}$. "NA" denotes that the approach is not applicable for the constraint. "_." denotes that no result is shown for the constraint in the approach. For d695, we observe that the proposed approach can achieve a $6.9 \%$ reduction in average test time compared to [14]. For h953, we observe that the proposed approach can achieve the lower bound (119357) on the SoC test time [8] under all power constraints. Moreover, in both SoCs under tight power constraints $\left(P_{\max }=1000\right.$ for d695, $P_{\max }=5 \times 10^{9}$ for $\left.\mathrm{h} 953\right)$, only the proposed method can provide a solution. This is because only the proposed method can lower the test frequencies and reduce the power consumption during test. From these results, we conclude that the proposed powerconscious virtual TAM technique and test scheduling algorithm are effective.

\section{Conclusions}

This paper has proposed a power-constrained test scheduling method for multi-clock domain SoCs. To the best of our knowledge, a test scheduling problem for multi-clock domain SoCs has been addressed and formulated for the first time in this paper. Moreover, we have proposed a technique to reduce power consumption of cores during test while the test time of the cores remain the same or increase a little by utilizing virtual TAMs. The experimental results showed that the proposed test scheduling method can achieve short test time compared to the previous power-constrained test scheduling methods.

\section{Acknowledgments}

This work was supported in part by Japan Society for the Promotion of Science (JSPS) under Grants-in-Aid for Scientific Research B(No. 15300018) and for Young Scientists B(No.18700046). The authors would like to thank Prof. Kewal K. Saluja, Prof. Michiko Inoue, Dr. Satoshi Ohtake and members of Computer Design and Test Laboratory in Nara Institute of Science and Technology for their valuable comments.

\section{References}

[1] Y. Zorian, E.J. Marinissen, and S. Dey, "Testing embedded-core based system chips," Proc. International Test Conference, pp.130143, Oct. 1998.

[2] "IEEE standard testability method for embedded core-based integrated circuits." IEEE Std 1500-2005, 2005.

[3] V. Iyengar, K. Chakrabarty, and E.J. Marinissen, "Test wrapper and test access mechanism co-optimization for system-on-chip,” J. Electron. Testing Theory and Appl. (JETTA), vol.18, no.2, pp.213-230, April 2002.

[4] W. Zou, S.R. Reddy, I. Pomeranz, and Y. Huang, "SOC test scheduling using simulated annealing," Proc. VLSI Test Symposium, pp.325-329, May 2003.

[5] E.J. Marinissen, S.K. Goel, and M. Lousber, "Wrapper design for embedded core test," Proc. International Test Conference, pp.911920, Oct. 2000.

[6] T. Ono, K. Wakui, H. Hikima, Y. Nakamura, and M. Yoshida, "Integrated and automated design-for-testability implementation for cellbased ICs," Proc. Asian Test Symposium, pp.122-125, Nov. 1997.

[7] P. Varma and S. Bhatia, "A structured test re-use methodology for core-based system chips,". Proc. International Test Conference, pp.294-302, Oct. 1998.

[8] S.K. Goel and E.J. Marinissen, "Effective and efficient test architecture design for SOC," Proc. International Test Conference, pp.529538, Oct. 2002.

[9] M. Nourani and C.A. Papachristou, "Structural fault testing of embedded cores using pipelining," J. Electron. Testing Theory and Appl. (JETTA), vol.15, no.1/2, pp.129-144, Aug.-Oct. 1999.

[10] S. Ravi, G. Lakshminarayana, and N.K. Jha, "Testing of core-based systems-on-a-chip," IEEE Trans. Comput.-Aided Des. Integr. Cir- 
cuits Syst., vol.20, no.3, pp.426-439, March 2001.

[11] T. Yoneda and H. Fujiwara, "Design for consecutive testability of system-on-a-chip with built-in self testable cores," J. Electron. Testing Theory and Appl. (JETTA), Special Issue on Plug-and-Play Test Automation for System-on-a-Chip, vol.18, no.4/5, pp.487-501, Aug. 2002.

[12] Y. Huang, W.T. Cheng, C.C. Tsai, N. Mukherjee, O. Samman, Y. Zaidan, and S.M. Reddy, "Resource allocation and test scheduling for concurrent test of core-based SOC design," Proc. Asian Test Symposium, pp. 265-270, Nov. 2001.

[13] V. Iyengar, K. Chakrabarty, and E.J. Marinissen, "On using rectangle packing for SOC wrapper/TAM co-optimization," Proc. VLSI Test Symposium, pp.253-258,, April 2002.

[14] Y. Huang, N. Mukherjee, S. Reddy, C. Tsai, W.T. Cheng, O. Samman, P. Reuter, and Y. Zaidan, "Optimal core wrapper width selection and SOC test scheduling based on 3-dimensional bin packing algorithm," Proc. International Test Conference, pp.74-82, Oct. 2002.

[15] Y. Xia, M.C. Jeske, B. Wang, and M. Jeske, "Using distributed rectangle bin-packing approach for core-based $\mathrm{SoC}$ test scheduling with power constraints," Proc. International Conference on ComputerAided Design, pp.100-105, Nov. 2003.

[16] E. Larsson, K. Arvidsson, H. Fujiwara, and Z. Peng, "Efficient test solutions for core-based designs," IEEE Trans. Comput.-Aided Des. Integr. Circuits Syst., vol.23, no.5, pp.758-775, May 2004.

[17] Q. Xu, Y. Zhang, and K. Chakrabarty, "SOC test architecture optimization for signal integrity faults on core-external interconnects," Proc. Design Automation Conference, pp.676-681, June 2007.

[18] A. Khoche, "Test resource partitioning for scan architectures using bandwidth matching," Digest of International Workshop on Test Resource Partitioning, pp.1.4-1-1.4-8, 2001.

[19] A. Sehgal, V. Iyengar, and K. Chakrabarty, "SOC test planning using virtual test access architectures," IEEE Trans. Very Large Scale Integr. (VLSI) Syst., vol.12, no.12, pp.1263-1276, Dec. 2004.

[20] Q. Xu and N. Nicolici, "Multi-frequency test access mechanism design for modular SOC testing," Proc. Asian Test Symposium, pp.27, Nov. 2004.

[21] Q. Xu and N. Nicolici, "Wrapper design for multi-frequency IP cores," IEEE Trans. Very Large Scale Integr. (VLSI) Syst., vol.13, no.6, pp.678-685, June 2005.

[22] Q. Xu, N. Nicolici, and K. Chakrabarty, "Multi-frequency wrapper design and optimization for embedded cores under average power constraints," Proc. Design Automation Conference, pp.123-128, June 2005.

[23] D. Zhao, U. Chandran, and H. Fujiwara, "Shelf packing to the design and optimization of a power-aware multi-frequency wrapper architecture for modular IP cores," Proc. Asia and South Pacific Design Automation Conference, pp.714-719, Jan. 2007.

[24] T.E. Yu, T. Yoneda, D. Zhao, and H. Fujiwara, "Using domain partitioning in wrapper design for IP cores under power constraints," Proc. VLSI Test Symposium, pp.369-374, May 2007.

[25] P. Girard, "Survey of low-power testing of VLSI circuits," IEEE Des. Test Comput., vol.19, no.3, pp.82-92, May/June 2002.

[26] K. Hatayama, M. Nakao, and Y. Sato, "At-speed built-in test for logic circuits with multiple clocks," Proc. Asian Test Symposium, pp.292-297, Nov. 2002.

[27] E.J. Marinissen, V. Iyengar, and K. Chakrabarty, "A set of benchmarks for modular testing of SOCs," Proc. International Test Conference, pp.519-528, Oct. 2002

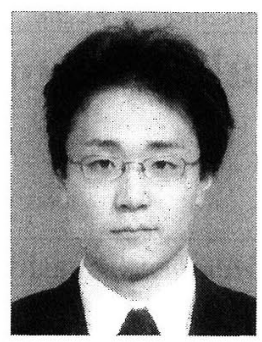

Tomokazu Yoneda received the B.E. degree in information systems engineering from Osaka University, Osaka, Japan, in 1998, and M.E. and $\mathrm{Ph} . \mathrm{D}$. degree in information science from Nara Institute of Science and Technology, Nara, Japan, in 2001 and 2002, respectively. Presently he is an assistant professor in Graduate School of Information Science, Nara Institute of Science and Technology. His research interests are VLSI CAD, design for testability and SoC testSociety. ing. He is a member of the IEEE Computer

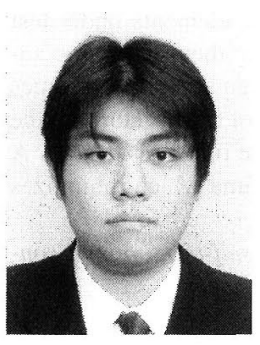

Kimihiko Masuda received the B.E. degree in engineering from Kyoto Institute of Technology, Kyoto, Japan, in 2003, and M.E. degree in information science from Nara Institute of Science and Technology, Nara, Japan, in 2005. Presently he works for SHARP Corporation.

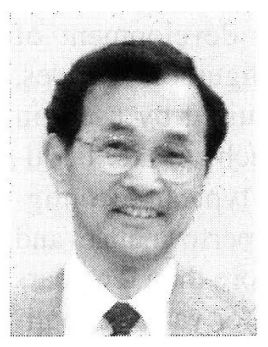

Hideo Fujiwara received the B.E., M.E., and Ph.D. degrees in electronic engineering from Osaka University, Osaka, Japan, in 1969, 1971, and 1974, respectively. He was with Osaka University from 1974 to 1985 and Meiji University from 1985 to 1993 , and joined Nara Institute of Science and Technology in 1993. In 1981 he was a Visiting Research Assistant Professor at the University of Waterloo, and in 1984 he was a Visiting Associate Professor at McGill University, Canada. Presently he is a Professor at the Graduate School of Information Science, Nara Institute of Science and Technology, Nara, Japan. His research interests are logic design, digital systems design and test, VLSI CAD and fault tolerant computing, including high-level/logic synthesis for testability, test synthesis, design for testability, built-in self-test, test pattern generation, parallel processing, and computational complexity. He is the author of Logic Testing and Design for Testability (MIT Press, 1985). He received the IEICE Young Engineer Award in 1977, IEEE Computer Society Certificate of Appreciation Award in 1991, 2000 and 2001, Okawa Prize for Publication in 1994, IEEE Computer Society Meritorious Service Award in 1996, and IEEE Computer Society Outstanding Contribution Award in 2001. He is an advisory member of IEICE Trans. on Information and Systems and an editor of IEEE Trans. on Computers, J. Electronic Testing, J. Circuits, Systems and Computers, J. VLSI Design and others. Dr. Fujiwara is a fellow of the IEEE, a Golden Core member of the IEEE Computer Society and a member the Information Processing Society of Japan. 